University of Rhode Island

DigitalCommons@URI

Cancer Prevention Research Center Faculty

Publications

Cancer Prevention Research Center

2012

\title{
Cluster subtypes within the precontemplation stage of change for sun protection behavior
}

\author{
Marimer Santiago-Rivas \\ University of Rhode Island \\ Wayne F. Velicer \\ University of Rhode Island, VELICER@URI.EDU \\ Colleen A. Redding \\ University of Rhode Island, credding@uri.edu \\ James O. Prochaska \\ University of Rhode Island, joprochaska@uri.edu \\ Andrea L. Paiva \\ University of Rhode Island, apaiva@uri.edu
}

Follow this and additional works at: https://digitalcommons.uri.edu/cprc_facpubs

This is a pre-publication author manuscript of the final, published article.

Terms of Use

All rights reserved under copyright.

\section{Citation/Publisher Attribution}

Santiago-Rivas, M., Velicer, W. F., Redding, C. A., Prochaska, J. O., \& Paiva, A. L. (2012). Cluster subtypes within the precontemplation stage of change for sun protection behavior. Psychology, Health \& Medicine, 17(3), 311-322. doi: 10.1080/13548506.2011.630401

Available at: https://doi.org/10.1080/13548506.2011.630401

This Article is brought to you for free and open access by the Cancer Prevention Research Center at DigitalCommons@URI. It has been accepted for inclusion in Cancer Prevention Research Center Faculty Publications by an authorized administrator of DigitalCommons@URI. For more information, please contact digitalcommons-group@uri.edu. 


\title{
Cluster subtypes within the precontemplation stage of change for sun protection behavior
}

\author{
Marimer Santiago Rivas ${ }^{a}, 1$, Wayne F. Velicer ${ }^{b}$, Colleen A. Redding ${ }^{b}$, James O. Prochaska ${ }^{b}$, \\ and Andrea L. Paiva ${ }^{a}$ \\ aDepartment of Psychology, University of Rhode Island, Kingston, USA \\ ${ }^{b}$ Cancer Prevention Research Center, Kingston, Rhode Island, United States
}

\section{Abstract}

Objective-The goal of this study is to identify replicable cluster subtypes within the Precontemplation stage of change for sun protection.

\begin{abstract}
Method-Secondary data analysis of baseline data from a sample of participants in a home-based expert system intervention was performed. Three random samples were selected from participants in the Precontemplation stage $(\mathrm{N}=570)$. Cluster analyses were performed using the scales of Pros, Cons and Self-Efficacy. Interpretability of pattern, pseudo F test, and dendograms were used to determine the number of clusters.
\end{abstract}

Results-A four-cluster solution replicated across subsamples. Significant differences between clusters on the nine Processes of Change, and on behavioral measures were found.

Discussion-Cluster solutions were robust, interpretable and with good initial external validity. They replicated patterns found for other behaviors, demonstrating long-term predictability and providing basis for tailored interventions.

\section{Keywords}

sun protection; clusters; prevention

Theories of behavior have used the concept of stages of change to represent the temporal and dynamic dimensions of behavior, and to make predictions about modification of behavior. The Transtheoretical Model of Behavior Change (TTM) has been applied to the study and modification of a range of health-related behaviors (Burbank, Reibe, Padula, \& Nigg, 2002; Fava, Velicer, \& Prochaska, 1995; Johnson et al., 2007; Norman et al., 2007; Prochaska et al., 1994, 2001, 2004, 2005; Weinstock, Rossi, Redding, \& Maddock, 2002). The main constructs are: Decisional Balance (Pros and Cons of behavior) and Temptations/ Self-Efficacy, temporal change of behavior (represented by stages of change), behavioral measures and outcomes, and processes of change.

Stage of change is the central organizing construct of the TTM approach. The five stages, Precontemplation, Contemplation, Preparation, Action, and Maintenance, represent the temporal dimension integrating current behavior and intention to engage in the new health behavior (Prochaska et al., 2005; Velicer et al., 2000). In the TTM, behavior change is visualized as a process that happens over time and that involves progression through these stages (Prochaska \& Norcross, 2001). As part of the assessment of the individual's stage of change, a staging algorithm is used. This algorithm uses specific time frames to determine

\footnotetext{
${ }^{1}$ Corresponding Author: Marimer Santiago-Rivas. smarimer@my.uri.edu.
} 
whether people plan a specific behavior change or not in the future. Particular time frames may be more or less suitable for different behaviors, settings, and samples. Other theories, such as the Health Action Process Approach (HAPA), also suggests the existence of phases or stages: non-intenders, intenders, and actors (Lippke, Ziegelmann, \& Schwarzer, 2005; Schwarzer, 2008). Craciun, Schüz, and Lippke (2011) reported that sun behavior variables typically used in the later phases of the HAPA (e. g., self-efficacy and strategic planning) mediate between intention to use sunscreen and sunscreen use. The HAPA model can predict future goal behavior, but critics have questioned some aspects of the theory, including use for development of interventions, and stage transition (Conner, 2008; Velicer \& Prochaska, 2008).

A limited number of distinct subtypes have been identified among smokers in the Precontemplation stage (Anatchkova et al., 2006a; Norman et al., 2000; Velice, Hughes, Fava, Prochaska, \& DiClemente, 1995; Velicer et al., 2007). Santiago-Rivas and colleagues (2010) examined profiles for sun protection within the Preparation stage, but no other study has indentified subtypes for sun protection within the Precontemplation stage.

\section{Present study}

This study was conducted in order to explore the existence of distinctive, interpretable, and internally consistent subgroup profiles within the Precontemplation stage using replication across three subsamples. Another aim of the study is to externally validate the clusters using the Processes of Change scales and sun protection behavior variables.

\section{Method}

\section{Participants}

The sample used in this secondary data analysis is a portion of a sample collected from a larger, multiple behavior intervention study of primary care patients. Outcome analyses are reported elsewhere (Prochaska et al., 2005). Participants were randomly assigned to either the home based expert system intervention or control condition. Only the treatment group was examined. The University's Institutional Review Board approved this study.

Eligibility for this study required being at risk for sun exposure. There were 570 participants classified in the Precontemplation stage of change at baseline. Most participants were female $(68.8 \%)$, White $(96.6 \%)$, reported good health status $(39.2 \%)$, and were married $(65.2 \%)$. The mean age was $47.46(S D=13.10)$.

\section{Measures}

Stages of Change-The general sun protection algorithm measures current behaviors, and sun protection intention (by avoiding sun exposure, using sunscreen, and/or wearing protective clothing). Participants in the Precontemplation stage don't consistently employ sun protection behaviors, and don't intend to start protecting themselves from exposure to the sun in a consistent manner in the future (they answered no to the following questions: (1) Do you protect yourself from exposure to the sun consistently, that is, whenever you know you will be out in the sun for more than about 15 minutes?; (2) Have you consistently protected yourself from exposure to the sun for the past 12 months?; (3) Do you intend to consistently protect yourself from exposure to the sun in the next 12 months?; and (4) Do you intend to consistently protect yourself from exposure to the sun in the next 30 days?)

Decisional Balance-The Decisional Balance Inventory derives from Janis and Mann's model of decision-making (Janis \& Mann, 1977). It measures two constructs, the Pros, or 
advantages related to the targeted behavior, and the Cons, or disadvantages related to the behavior (Velicer, DiClemente, Prochaska, \& Brandenberg, 1985). The instrument used in this study consists of 4 items assessing the Pros of sun protection $(a=0.78)$ and 4 items assessing the Cons of sun protection ( $a=0.74)$ (Maddock et al, 2005; Prochaska et al., 1994). It uses a 5-point Likert scale from non - important $(=1)$ to extremely important $(=5)$.

Self-Efficacy-The original Self Efficacy measure was developed and verified with adult smokers (Velicer, DiClemente, Rossi, \& Prochaska, 1990). This is a measure was based on the self-efficacy construct of Bandura $(1977 ; 1982)$ and the relapse prevention literature. It consists of 7 items measuring confidence to use sun protection $(\alpha=0.84)$, and uses a 5-point Likert scale from not at all confident $(=1)$ to extremely confident $(=5)$ (Maddock et al., 2005).

Processes of Change-These processes are the independent variables that people need to apply, or be engaged in, to progress from stage to stage (Prochaska, Velicer, DiClemente, \& Fava, 1988; Velicer et. al., 2000). Nine of the 10 traditional processes of change were assessed using 2 items each. The processes included counter conditioning, consciousness raising, dramatic relief, environmental reevaluation, helping relationships, reinforcement management, self reevaluation, social liberation, and self-liberation. Respondents were asked how often in the past 30 days they used the processes of change on a 5- point Likert scale (never $=1$ to always $=5$ ). Coefficient alphas ranged from 0.71 to 0.81 in previous studies (Maddock et al., 2005).

Behavioral Measures-The Sun Protection Behavior Scale (SPBS) is a brief inventory with three subscales that include measures of sun protection (Weinstock et al., 2000). Two subscales were used: Sunscreen Use $(a=0.86)$, and Sun Avoidance $(a=0.82)$. Each of the seven items is a self-report of behavior on a 5-point Likert scale of frequency (never, rarely, sometimes, often, and always) "when in the sun for more than about 15 minutes during the summer". The total score of this scale was used. Additional variables employed as validity measures for clustering solutions included: use of sunscreen, SPF, and tanning booth use history in both the past generally ("have you ever used a tanning booth or sunlamp") and in the previous year ("have you used a tanning booth or sunlamp in the past year").

\section{Procedure}

Three subsamples were formed: Sample $1(\mathrm{~N}=182 ; 8$ participants were not clustered due to incomplete information), Sample 2 ( $N=187 ; 3$ participants were not clustered) and Sample 3 ( $\mathrm{N}=186 ; 4$ participants were not clustered). Cluster analysis was performed within each sample independently, and with the overall sample. Pros, Cons, and Self-efficacy scores were used to identify the profiles.

\section{Cluster Analysis}

Similarity measures have been developed to estimate the similarity or proximities, between the individuals. The most commonly used is the squared Euclidean distance (Everitt, Landau, \& Leese, 2001), which was employed in this study. The squared Euclidean distance metric was calculated on the Pros, Cons, and Self-efficacy. Ward's minimum variance method (Ward, 1963) was employed to form the cluster. Several indices were used to determine the number of clusters: the cubic clustering criterion (CCC), the pseudo $F$ test, and the pseudo $t^{2}$ (Calinski \& Harabasz, 1974; SAS Institute Inc., 1999). Following the results from the three indices, visual inspection of the cluster profiles was performed. The variables to be included in cluster identification procedures were standardized within stages to $T$ - scores $(M=50, S D=10)$. 


\section{Results}

Four clusters were initially found to replicate among the three subsamples. This initial solution was applied to the general sample. The cluster profiles for the combined sample ( $\mathrm{N}$ $=555)$ are presented in Figure 1 and these clusters were used for the external validity. The clusters are described below:

Cluster 1: The first cluster ( $\mathrm{N}=142 ; 25.59 \%$ of participants) was labeled Immotive, and was characterized by an inverted "V" shape with medium scatter and average level. This profile corresponds to what is expected for people in the Precontemplation stage. The means for Pros and Self-efficacy scores were below average, while the mean for the Cons scale was above average. Participants in this group consider the Cons of protecting themselves from sun exposure much more important than the Pros. This cluster was the most stable across subsamples.

Cluster 2: The second cluster ( $\mathrm{N}=197 ; 35.49 \%$ of participants) was labeled Progressing, and had a "V" shape with high scatter and above average level. This subgroup had average scores on the Cons scale, and above average scores on the Pros and Self-efficacy scales. This pattern is similar to what would be found in an advanced stage, and individuals may be getting ready to progress to a more advanced stage. They are considering both the benefits and the disadvantages of acquiring healthier sun protection habits. Self-efficacy scores are higher than what would be expected from people that are not currently practicing sun protection. This was the largest cluster.

Cluster 3: The third cluster ( $\mathrm{N}=57 ; 10.27 \%$ of participants) was labeled Disengaged and had a slightly inverted "V" shape, low to medium scatter, and low level. This subtype consists of participants with Pros, Cons, and Self-efficacy scores all about a standard deviation below average. Members did not appear to feel strongly about sun protection or sun exposure. They are reporting no positive or negative views about sun protection, and low self-efficacy. This was the smallest group.

Cluster 4: The fourth cluster ( $\mathrm{N}=159 ; 28.65 \%$ of participants) was labeled Early Progressing and had a shallow "V" shape (almost a flat shape) with medium scatter. The mean for the Self-efficacy scale was about average, while the scores for the Pros and Cons scales were below average. This indicates a very minimal engagement with the conscious acquisition of sun protective behaviors. Individuals in this group were minimizing the positive aspects of sun protection. This was the second largest group overall.

\section{External validation: Processes of change}

A Multivariate Analysis of Variance was performed using cluster membership as the grouping variable and significant multivariate differences were found for the 9 of the 10 processes (Wilks's $=.591, \mathrm{p}<.001, \eta 2=.409$ ). Descriptive statistics, univariate F values, Tukey patterns and values for partial eta-squared for the clusters are presented in Table 1. The effect sizes ranged from small to large. The Immotive cluster was the subtype that most resembled the expected profile for Precontemplation. This group used these processes in the medium range, showing a profile similar to the one reported by the Early Progressing cluster. The Progressing cluster had almost the opposite profile of the Immotive subtype. Members of the Progressing cluster reported using all the processes the most. The Early Progressing cluster used these processes in the medium range, showing a profile similar to the one reported by the Immotive cluster on 6 of the 9 processes. The Disengaged cluster used the process the least. 


\section{External validation: Behavioral variables}

A significant difference between clusters was found on scores of the Sun Protection Behavior Scale (SPBS), F $(3,551)=64.63, \mathrm{p}<.001, \eta 2=.256$ (see Table 2). The effect size for this measure was large, and the follow up Tukey test revealed that participants in the Progressing cluster reported doing the most to protect their skin from the sun compared to the other clusters, while members of the Immotive and Disengaged clusters reported doing the least. The Early Progressing subtype reported a relatively high score on the SPBS.

Chi-square tests were performed on the other behavioral variables (see Table 3). Significant differences were found for sunscreen use a, $\chi 2(15)=107.58, \mathrm{p}<.001$; Cramer's $\Phi^{2}=.258$. A higher percentage of participants in the Disengaged cluster $(75 \%)$ reported never using sunscreen, while a lower percentage of participants in the Progressing group (11.6\%) reported never using sunscreen. Members of the Immotive cluster reported similar behavioral profile as the Early Progressing cluster. In terms of SPF of sunscreen, a higher percentage of members of the Progressing and Early Progressing clusters (43.2\% and 34.8\%, respectively) reported using sunscreen with a protection factor of 15 to 29 , in comparison to participants in the Immotive and Disengaged clusters (20\% and 7.7\%, respectively). Even when all participants responded that they don't protect themselves from exposure to the sun consistently, results show that they still report some occurrence of sun protection behavior.

The respondents were asked to report if they ever used artificial tanning/sun lamp in the past, and if yes, to report use during the previous year (see Table 3 ). Chi-square tests found significant differences for ever use, $\chi 2(15)=37.42, p<.001$; Cramer's $\Phi^{2}=.260$, and previous year $\chi^{2}(15)=8.99, \mathrm{p}<.05$; Cramer's $\Phi^{2}=.198$. Most members of the Disengaged cluster reported that they'd visited a tanning booth or used a sun lamp at least once in their lives (87.7\%), while around 50\% of the Immotive and Progressing clusters reported they'd done so. Among those that reported artificial tanning use, 50.7\% of the Immotives reported use at least once during the past year. This cluster showed a low exercise of sun protection behaviors. The rest of the clusters were characterized by a lower prevalence of artificial tanning.

\section{Discussion}

One of the main findings of this exploratory study is the identification and replication of a four-cluster solution across three samples within the Precontemplation stage for sun protection. The similarity of the subgroup profiles based on the scores from the Pros, Cons, and Self-efficacy was evaluated. Replication is critical for any cluster analysis study since the cluster analysis is an exploratory method that will always produce clusters. If there are no natural clusters, the methods results in 'dissection' (Everitt, Landau, \& Leese, 2001). If natural clusters exist, they should demonstrate internal cohesion (homogeneity) and external isolation (separation). Replication provides critical evidence that the correct number of clusters has been selected and can help guide the interpretation of the clusters. In this case, replication occurred not only within the samples for sun protection but also replicates results reported for another behavior (for smoking; see Anatchkova et al., 2006a; Norman et al., 2000; Velicer et al., 1995; Velicer et al., 2007).

The processes of sun protection varied significantly across profiles within the Precontemplation stage. As expected, the Progressive profile used the processes the most, while the Disengaged group used them the least. The Progressive cluster reported higher scores across processes in comparison to the rest of the subtypes, with the exception of Social Liberation ("I notice that many people are protecting themselves from the sun these days"; "I see more and more people using sunscreens to protect themselves from the sun"), Counter Conditioning ("I do something else instead of sun bathing when I need to relax"; 
"Instead of tanning, I do other things"), and Helping Relationships ("People who are important to me encourage me to protect myself from the sun"; "I have a friend or family member who reminds me to use sunscreen"). The Immotive and Early Progressing subtypes reported similar scores, with the exception of Social Liberation and Counter Conditioning. Differences in the use of these processes seem to be less evident across clusters, and smaller effect sizes were reported for these processes. As in previous studies where the use of processes by stages and clusters is examined, Social Liberation (and Environmental Reevaluation) accounted for less variance in comparison to other cognitive processes. This suggests that these processes are less relevant for the participants (Anatchkova et al., 2006; Fava et al., 1995; Norman et al., 2000; Velicer et al., 1995). A similar outcome was found for Helping Relationships, which is a behavioral process. The Early Progressing and Immotive groups became less similar when other variables, such as use of sunscreen and history of artificial tanning, were considered.

\section{Limitations}

Cluster analysis is an exploratory approach that employs the interpretation of the researcher in the identification of the subgroups. In this study, replication across three samples and external validation using variables not included in the clustering process control for the influence of experimenter bias on the results.

Even when the general sample used for the final results was large, the subsamples were small. This may have affected the stability of the clusters and it influences the confidence in the results. In addition, this sample of primary care patients was primarily White (thus not representative of other ethnic and racial groups), female, and middle-aged. The sample was recruited from a subset of practices participating in an educational outreach intervention to promote practice adoption of cancer prevention practices (Prochaska et al., 2005). If practices enrolled in the study endorse more cancer prevention activities than others, application of the results may be limited. Weinstock and colleagues (2000; 2002) reported that, in a sample of beachgoers in the New England area, increased age and female gender were associated with more sun protective behaviors. This suggests that different patterns of baseline behavior would be expected in men, or in other ager groups. These factors are important when assessing the suitability of interventions for dissemination.

Another limitation of the study is that it focuses on a single stage. But additional analyses on the Contemplation stage will be presented in a separate research report. Analyses on the Preparation stage are published elsewhere (Santiago-Rivas et al., 2010).

\section{Utility of clusters}

Everitt, Landau, and Leese (2001) provide a description of the many reasons for developing a classification system and provide numerous examples from different branches of science. Of particular interest in the area of health promotion is that the identification of cluster subtypes has the potential to guide the development of tailored interventions for the promotion of sun protection habits. Tailored interventions have typically been based on developed by employing a serious of variables identified as critical to success and then using those variables sequentially to subdivide the sample (Velicer et al., 1993; Velicer \& Prochaska, 1999). An alternative method is to use the cluster subgroups as the basis for tailoring and assign individuals to groups based on their distance from each of the subgroups (Levesque, Driskell, \& Prochaska, 2008). There is no evidence about which approach is the most effective. The clusters identified in this study are of special interest because traditionally individuals in the Precontemplation stage present the most serious challenge for the promotion of healthy behaviors and for the prevention of risk behaviors. In addition, the use of clusters helps in the identification of information that might be more relevant to some 
participants than others. In some studies that examined the HAPA approach, the number of participants in the advanced stage of this model was significantly greater in comparison to the rest of the stages (Duan, Lippke, Wagner, \& Brehm, 2010; Lippke et al., 2005; Lippke, Fleig, Pomp, \& Schwarzer, 2010). Other studies classified participants in two stages instead of three (Schüz, Sniehotta, \& Schwarzer, 2007; Renner \& Schwarzer, 2005). These aspects affect the generalization and application of the findings. Studies that applied the TTM to develop stage-based interventions are largely extended to representative at-risk populations (Prochaska et al., 1994, 2001, 2004, 2005; Weinstock et al., 2002).

The existence of these profiles should not be considered as additional stages to the stages of change. They provide an alternative course of action to potential interventions. They support the current 5 stages included in the TTM (Velicer et al., 1995), and provide complementary information about sun protection behavior and relevant processes. Subtypes were replicated, and they are interpretable within the conceptualization of the model used in this study. Considerations like the cost-effectiveness, purpose, length, sample, and sample size of an intervention may make the use of subtypes more or less appropriate to intervene for a behavior problem.

\section{Acknowledgments}

Grants 71356, Grants CA50087 CA119195, AG024490, and CA27821 from the National Cancer Institute supported this research.

\section{References}

Aldenderfer, MS.; Blashfield, RK. Cluster analysis. Beverly Hills, CA: Sage Publications; 1984.

Anatchkova MD, Velicer WF, Prochaska JO. Replication of subtypes for smoking cessation within the contemplation stage of change. Addictive Behaviors. 2005; 30:915-927. [PubMed: 15893089]

Anatchkova MD, Velicer WF, Prochaska JO. Replication of subtypes for smoking cessation within the precontemplation stage of change. Addictive Behaviors. 2006a; 31:1101-1115. [PubMed: 16139436]

Anatchkova MD, Velicer WF, Prochaska JO. Replication of subtypes for smoking cessation within the preparation stage of change. Addictive Behaviors. 2006b; 31:359-366. [PubMed: 15967587]

Bandura A. Self-efficacy: Toward a unifying theory of behavior change. Psychological Review. 1977; 84:191-215. [PubMed: 847061]

Bandura A. Self-efficacy mechanisms in human agency. American Psychologist. 1982; 37:122-147.

Burbank PM, Reibe D, Padula CA, Nigg C. Exercise and older adults: Changing behavior with the transtheoretical model. Orthopaedic Nursing. 2002; 21 (4):51-62. [PubMed: 12224187]

Calinski RB, Harabasz J. A dendrite method for cluster analysis. Communication in statistics. 1974; $3: 1-27$.

Craciun C, Schüz N, Lippke S. A mediator model of sunscreen use: A longitudinal analysis of socialcognitive predictors and mediators. International Journal of Behavioral Medicine, Online First. 2011

Conner M. Initiation and maintenance of health behaviors. Applied Psychology: An International Review. 2008; 57:42-50.

Duan Y, Lippke S, Wagner P, Brehm W. Testing two stage assessments in a Chinese college student sample: Correspondences and discontinuity patterns across stages. Psychology of Sports and Exercise. 2011; 12:306-313.

Everitt, BS.; Landau, S.; Leese, M. Cluster Analysis. 4. New York: Oxford Univ. Press; 2001.

Fava JL, Velicer WF, Prochaska JO. Applying the Transtheoretical model to a representative sample of smokers. Addictive Behaviors. 1995; 20:189-203. [PubMed: 7484313]

Janis, IL.; Mann, L. Decision making: A psychological analysis of conflict, choice and commitment. New York: Free Press; 1977. 
Johnson SS, Paiva AL, Cummins CO, Johnson JL, Dyment SJ, Wright JA, Sherman K. Transtheoretical model-based multiple behavior intervention for weight management: Effectiveness on a population basis. Preventive Medicne. 2007; 46:238-246.

Levesque DA, Driskell MM, Prochaska JM. Acceptability of a stage-matched expert system intervention for domestic violence offenders. Violence and Victims. 2008; 23:432-445. [PubMed: 18788337]

Lippke S, Fleig L, Pomp S, Schwarzer R. Validity of a stage algorithm for physical activity in participants recruited from orthopedic and cardiac rehabilitation clinics. Rehabilitation Psychology. 2010; 4:398-408. [PubMed: 21171799]

Lippke S, Ziegelmann JP, Schwarzer R. Stage-specific adoption and maintenance of physical activity: Testing a three-stage model. Psychology of Sport and Exercise. 2005; 6:585-603.

Maddock JE, Redding CA, Rossi JS, Weinstock MA. Development and validation of an appearance motivation attitudes scale for sun protection. Psychology and Health. 2005; 20:775-788.

Norman GJ, Velicer WF, Fava JL, Prochaska JO. Cluster subtypes within stage of change in a representative sample of smokers. Addictive Behaviors. 2000; 25:183-204. [PubMed: 10795944]

Norman GJ, Adams MA, Calfas KJ, Covin J, Sallis JF, Rossi JS, Patrick K. A randomized controlled trial of a multicomponent intervention for adolescent sun protection behaviors. Archives of Pediatric \& Adolescent Medicine. 2007; 161:146-152.

Prochaska JO, Norcross JC. Stages of change. Psychotherapy. 2001; 38:443-448.

Prochaska JO, Velicer WF, DiClemente CC, Fava JL. Measuring the processes of change: Applications to the cessation of smoking. Journal of Consulting and Clinical Psychology. 1988; 56:520-528. [PubMed: 3198809]

Prochaska JO, Velicer WF, Fava JL, Ruggiero L, Laforge RG, Rossi JS, Lee PA. Counselor and stimulus control enhancements of a stage-matched expert system intervention for smokers in a managed care setting. Preventive Medicine. 2001; 32:23-32. [PubMed: 11162323]

Prochaska JO, Velicer WF, Redding C, Rossi JS, Goldstein M, DePue J, Plummer BA. Stage-based expert systems to guide a population of primary care patients to quit smoking, eat healthier, prevent skin cancer, and receive regular mammograms. Preventive Medicine. 2005; 41:406-416. [PubMed: 15896835]

Prochaska JO, Velicer WF, Rossi JR, Goldstein MG, Marcus BH, Rakowski W, Rossi SR. Stages of change and decisional balance for 12 problem behaviors. Health Psychology. 1994; 13:39-46. [PubMed: 8168470]

Prochaska JO, Velicer WF, Rossi JS, Redding CA, Greene GW, Rossi SR, Plummer BA. Multiple risk expert system interventions: Impact of simultaneous stage-matched expert system interventions for smoking, high-fat diet, and sun exposure in a population of parents. Health Psychology. 2004; 25:503-516. [PubMed: 15367070]

Renner B, Schwarzer R. The motivation to eat a healthy diet: How intenders and nonintenders differ in terms of risk perception, outcome expectancies. Polish Psychological Bulletin. 2005; 36:7-15.

Santiago-Rivas M, Velicer WF, Redding CA, Prochaska JO, Paiva AL. Cluster subtypes within the preparation stage of change for sun protection behavior. Applied Psychology: Health and Well Being. 2010; 2(1):89-104.

SAS Institute, Inc. SAS/STAT User's Guide, Version 8. Cary, NC: Author; 1999.

Schüz B, Sniehotta FF, Schwarzer R. Stage-specific effects of an action control intervention on dental flossing. Health Education Research. 2007; 22:332-341. [PubMed: 16945985]

Schwarzer R. Modeling health behavior change: How to predict and modify the adoption and maintenance of health behaviors. Applied Psychology: An International Review. 2008; 57:1-29.

Velicer WF, DiClemente CC, Prochaska JO, Brandenberg N. A decisional balance measure for assessing and predicting smoking status. Journal of Personality and Social Psychology. 1985; 48:1279-1289. [PubMed: 3998990]

Velicer WF, DiClemente C, Rossi JS, Prochaska JO. Relapse situations and self-efficacy: An integrative model. Addictive Behaviors. 1990; 15:271-283. [PubMed: 2378287]

Velicer WF, Hughes SL, Fava JL, Prochaska JO, DiClemente C. An empirical typology of subjects within stage of change. Addictive Behaviors. 1995; 20:299-320. [PubMed: 7653313] 
Velicer WF, Prochaska JO. An expert system intervention for smoking cessation. Patient Education and Counseling. 1999; 36:119-129. [PubMed: 10223017]

Velicer WF, Prochaska JO. Stage and non-stage theories of behavior and behavior change: A comment on Schwarzer. Applied Psychology: An International Review. 2008; 57:75-83.

Velicer WF, Prochaska JO, Bellis JM, DiClemente CC, Rossi JS, Fava JL, Steiger JH. An expert system intervention for smoking cessation. Addictive Behaviors. 1993; 18:269-290. [PubMed: 8342440]

Velicer WF, Prochaska JO, Fava JL, Rossi JS, Redding CA, Laforge RG, Robbins ML. Using the transtheoretical model for population based approaches to health promotion and disease prevention. Homeostasis in Health and Disease. 2000; 40:174-195.

Velicer WF, Redding CA, Anatchkova MD, Fava JL, Prochaska JO. Identifying cluster subtypes for the prevention of adolescent smoking acquisition. Addictive Behaviors. 2007; 32:228-247. [PubMed: 16697533]

Ward JH. Hierarchical grouping to optimize an objective function. Journal of the American Statistical Association. 1963; 58:236-244.

Weinstock MA, Rossi JS, Redding CA, Maddock JE. Randomized controlled community trial of the efficacy of a multicomponent stage-matched intervention to increase sun protection among beachgoers. Preventive Medicine. 2002; 35:548-592. [PubMed: 12460522]

Weinstock MA, Rossi JS, Redding CA, Maddock JE, Cottrill SD. Sun protection behaviors and stages of change for the primary prevention of skin cancers among beachgoers in southeastern New England. Annals of Behavioral Medicine. 2000; 22:286-293. [PubMed: 11253439] 


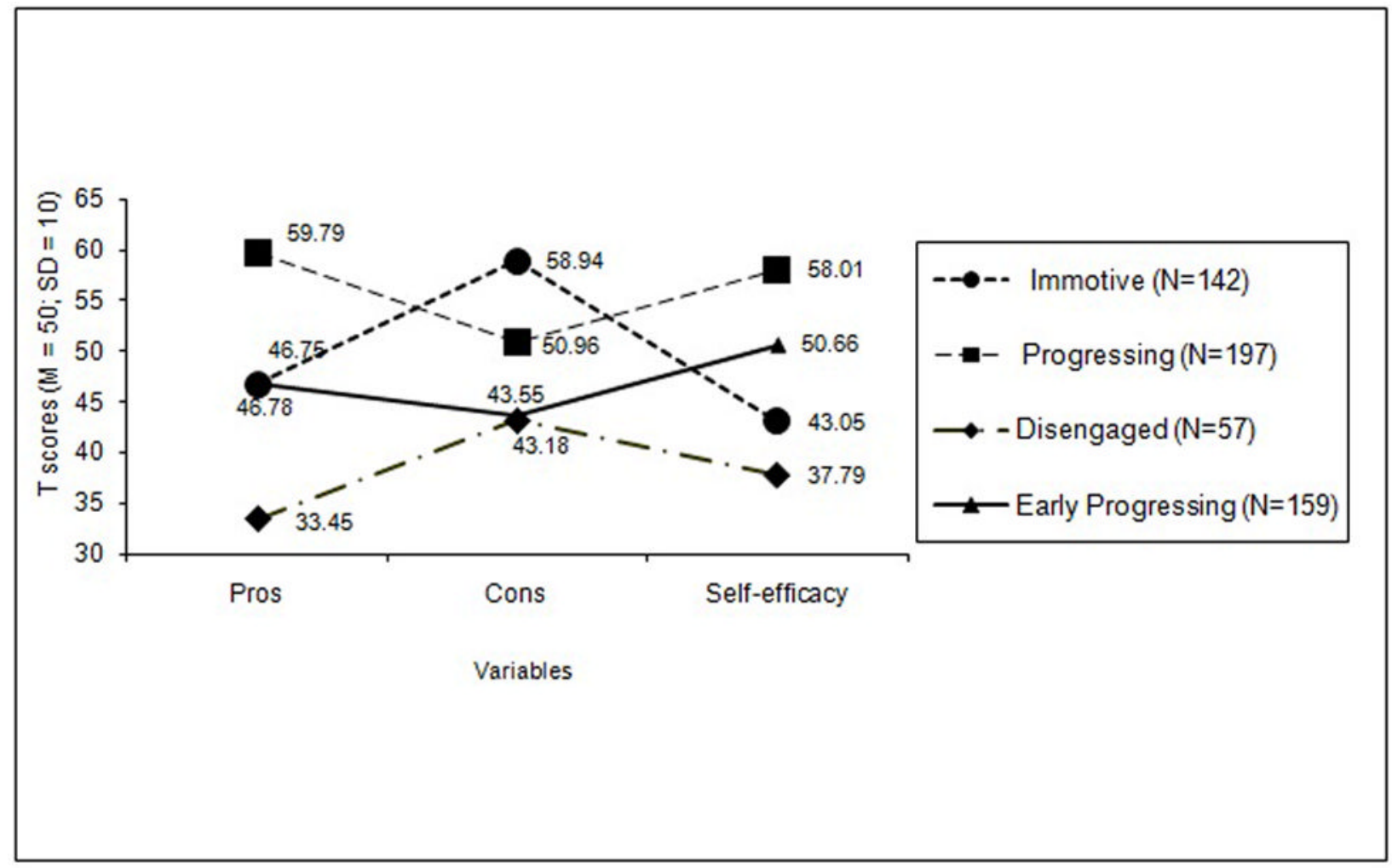

Figure 1.

Subtypes for the precontemplation stage from the general sample. 
Table 1

Means, standard deviations and subtypes comparisons for sun protection processes of change.

\begin{tabular}{|c|c|c|c|}
\hline Processes & $\mathbf{M}$ & SD & \\
\hline Consciousness Raising & & & $\mathrm{F}=43.09^{* *} ; \eta 2=.186$ \\
\hline Early Progressing & 4.65 & 1.46 & \\
\hline Progressing & 5.93 & 1.77 & $\mathrm{P}>$ all \\
\hline Immotive & 4.59 & 1.57 & $\mathrm{I}, \mathrm{E}>\mathrm{D}$ \\
\hline Disengaged & 3.51 & 1.65 & \\
\hline Dramatic Relief & & & $\mathrm{F}=50.80^{* * *} ; \eta 2=.213$ \\
\hline Early Progressing & 5.35 & 1.99 & \\
\hline Progressing & 7.31 & 2.04 & $\mathrm{P}>$ all \\
\hline Immotive & 5.63 & 1.82 & $\mathrm{I}, \mathrm{E}>\mathrm{D}$ \\
\hline Disengaged & 4.28 & 1.80 & \\
\hline Environmental Reevaluation & & & $\mathrm{F}=40.50^{* *} ; \eta 2=.176$ \\
\hline Early Progressing & 5.23 & 1.69 & \\
\hline Progressing & 6.62 & 1.95 & $\mathrm{P}>$ all \\
\hline Immotive & 5.10 & 1.76 & $\mathrm{I}, \mathrm{E}>\mathrm{D}$ \\
\hline Disengaged & 4.04 & 1.85 & \\
\hline Self Re-evaluation & & & $F=71.62^{* *} ; \eta 2=.277$ \\
\hline Early Progressing & 4.52 & 1.90 & \\
\hline Progressing & 6.53 & 2.10 & $\mathrm{P}>$ all \\
\hline Immotive & 4.51 & 1.89 & $\mathrm{I}, \mathrm{E}>\mathrm{D}$ \\
\hline Disengaged & 2.82 & 1.36 & \\
\hline Social Liberation & & & $\mathrm{F}=8.84^{* *} ; \eta 2=.041$ \\
\hline Early Progress & 7.10 & 1.58 & \\
\hline Progressing & 7.82 & 1.64 & $\mathrm{P}>\mathrm{E}, \mathrm{D}$ \\
\hline Immotive & 7.43 & 1.65 & $\mathrm{I}>\mathrm{D}$ \\
\hline Disengaged & 6.75 & 1.93 & \\
\hline Counter Conditioning & & & $\mathrm{F}=9.48^{* *} ; \eta 2=.044$ \\
\hline Early Progressing & 7.16 & 2.23 & \\
\hline Progressing & 7.05 & 2.00 & $\mathrm{P}, \mathrm{E}>\mathrm{I}$ \\
\hline Immotive & 5.98 & 2.32 & $\mathrm{E}>\mathrm{D}$ \\
\hline Disengaged & 6.19 & 2.87 & \\
\hline Helping Relationship & & & $\mathrm{F}=13.67^{* *} ; \eta 2=.064$ \\
\hline Early Progressing & 5.23 & 2.34 & \\
\hline Progressing & 6.13 & 2.40 & $\mathrm{P}>\mathrm{E}, \mathrm{D}$ \\
\hline Immotive & 5.91 & 2.36 & $\mathrm{E}, \mathrm{I}>\mathrm{D}$ \\
\hline Disengaged & 4.04 & 2.38 & \\
\hline Reinforcement Management & & & $\mathrm{F}=11.74^{* *} ; \eta 2=.055$ \\
\hline Early Progressing & 2.65 & 0.99 & $\mathrm{P}>$ all \\
\hline Progressing & 3.04 & 1.29 & $\mathrm{I}, \mathrm{E}>\mathrm{D}$ \\
\hline
\end{tabular}




\begin{tabular}{llll} 
Processes & M & SD & \\
\hline Immotive & 2.64 & 0.99 & \\
Disengaged & 2.16 & 0.45 & \\
Self-Liberation & & & $\mathrm{F}=54.45^{* *} ; \eta 2=.217$ \\
Early Progressing & 4.00 & 1.63 & $\mathrm{P}>$ all \\
Progressing & 5.28 & 1.92 & $\mathrm{I}, \mathrm{E}>\mathrm{D}$ \\
Immotive & 3.54 & 1.50 & \\
Disengaged & 2.63 & 1.13 & \\
\hline
\end{tabular}

E= Early Progressing, $\mathrm{P}=$ Progressing, I= Immotive, $\mathrm{D}=$ Disengaged; ** $p<.01$ 
Table 2

Means, Standard Deviations, and Subtype Comparisons for the Sun Protection Behavior Scale.

\begin{tabular}{|c|c|c|c|}
\hline Sun Protection Behavior Scale & $\mathbf{M}$ & SD & \\
\hline & & & $\mathrm{F}=64.63^{* *} ; \eta 2=.260$ \\
\hline Early Progressing & 17.57 & 4.39 & $\mathrm{P}>$ all \\
\hline Progressing & 20.54 & 1.77 & $\mathrm{E}>\mathrm{I}, \mathrm{D}$ \\
\hline Immotive & 14.44 & 4.48 & \\
\hline Disengaged & 13.44 & 3.69 & \\
\hline
\end{tabular}




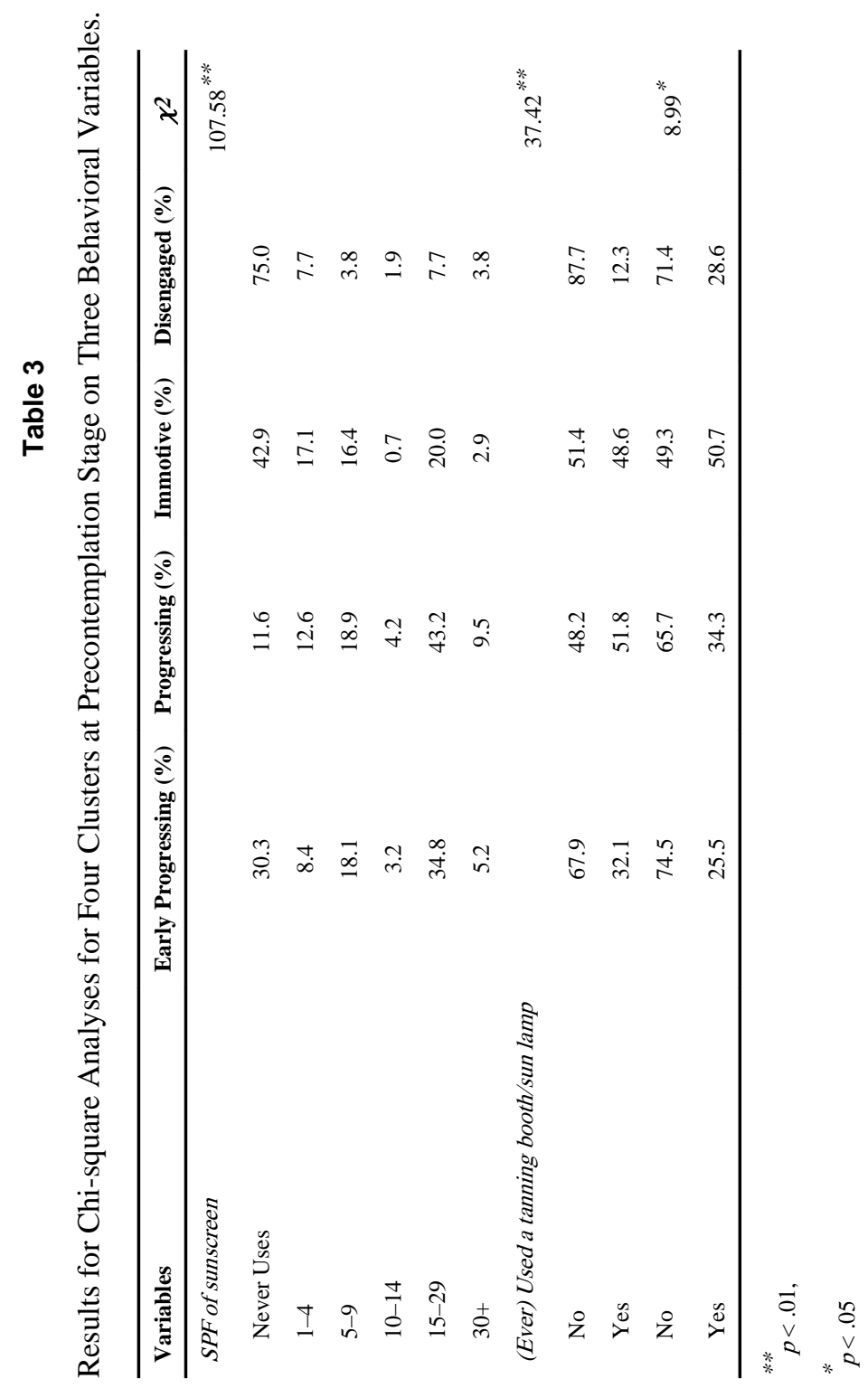

Psychol Health Med. Author manuscript; available in PMC 2013 May 01. 